\title{
Numerical Simulations of the UCLA/LANL/RRCKI/SLAC Experiment on a High Gain SASE FEL
}

\author{
E.L. Saldin ${ }^{\mathrm{a}}$, E.A. Schneidmiller ${ }^{\mathrm{a}}$ and M.V. Yurkov ${ }^{\mathrm{b}}$ \\ a Automatic Systems Corporation, 443050 Samara, Russia \\ boint Institute for Nuclear Research, Dubna, 141980 Moscow Region, Russia
}

\begin{abstract}
In this paper we present theoretical analysis of a recent SASE FEL experiment performed by UCLA/LANL/RRCKI/SLAC team reporting on a high power gain of about $10^{5}$ at the wavelength of $12 \mu \mathrm{m}$ [1]. The region of physical parameters of this experiment (as well as of future X-ray FELs) does not allow to apply available analytical techniques for quantitative description of the obtained results. The analysis presented in this paper is based on the results produced by a threedimensional, time-dependent FEL simulation code S\&D-3T [2]. It is shown that within the limit of accuracy of the experiment obtained data fully agree with the results of numerical simulations. Statistical simulations of the energy fluctuations in the radiation pulse performed over several thousands shots give the result identical to the experimental one. Namely, fluctuations of the energy in the radiation pulse follow gamma distribution with the value of parameter $M \simeq 8$. We also predict specific behaviour of another characteristics of the SASE FEL which can be measured during further experiments.
\end{abstract}




\section{Introduction}

Discussion on the possibility to generate powerful coherent VUV and X-ray radiation in a single-pass FEL amplifier starting from noise started in early 80 th [3, 4]. At present such devices (named as Self $\Lambda$ mplified Spontancous Emission (SASE) FEL [5]) arc planned for construction at several laboratories [6] - [1]]. Optimization of parameters of these devices requires development of three-dimensional, time-dependent theory of the FEL amplifier. Unfortunately, analytical techniques do not provide reliable tools for quantitative description of the obtained results. Some averaged output characteristics of SASE FEL have been obtained in refs. $[3,5,12,13,14,15,16,17]$. It has. been shown recently that the radiation from SASE FEL operating in the linear regime possesses all the features corresponding to completely chaotic polarized radiation. In particular, the higher order correlation functions (time and spectral) are expressed via the first order correlation function, the probability density distribution of the instantaneous radiation prwer follows the negative exponential distribution and the probability density function of the finite-time integrals of the instantaneous power and of the energy after monochromator follows the gamma distribution [18, 19]. An approach for time-dependent numerical simulations of SASE FEL radiation has been developed in ref. [17]. Realization of this approach allowed to obtain some statistical properties of the radiation from SASE FEL operating in linear and nonlinear regime $[17,20,21,18,19]$. Recently simulation code GINGER has been upgraded for simulation of the start-up from noise [22]. The works on construction of reliable and fast codes are under the way in several laboratories.

Recently it has been reported on an operation of a high gain FEL amplifier starting from noise [1] (see Table 1). The power gain of larger than $10^{5}$ has been obtained. Parameters of the output radiation have been measured with a high accuracy. In particular, it has been demonstrated that the fluctuations of the energy in the radiation pulse follows the gamma distribution predicted in ref. [18]. Thorough analysis shows that despite this experiment has been performed at a relatively long wavelength, the physical processes in this SASE FEL are similar to those expected to occur in a short wavelength SASE FEL. This is connected with the fact that the aperture of the vacuum chamber is significantly larger than the transverse size of the beam radiation mode. As a result, the waveguide effects do not influence the amplification process. Also, the effects of coherent synchrotron radiation are not important at the chosen parameters of the experiment. Helpful factor simplifying analysis of this experiment consists in careful choice of experimental conditions. Namely, undulator field has been tuned precisely and distortions of the electron beam trajectory are significantly less than typical transverse size of the beam radiation mode. There is also significant safety margin with respect to the energy spread and the emiitance of the electron beam. 
Table 1. Parameters of the UCLA/LANL SASE FEL [1]

\begin{tabular}{ll} 
Electron bearn & \\
\hline Energy [MeV] & 18 \\
Charge per micropulse $[\mathrm{nC}]$ & $0.3-2.2$ \\
Transverse spot size $(\sigma)[\mu \mathrm{m}]$ & $115-145$ \\
Energy spread $(\mathrm{rms})[\%]$ & $\sim 0.3$ \\
Pulse length (FWHM) [ps] & $7-13$ \\
Peak current [A] & $40-170$ \\
Undulator & \\
\hline Period [cm] & 2.05 \\
Number of periods & 98 \\
Undulator parameter $K$ & 1 \\
Betatron wavelength [m] & 1.2 \\
FEL & 12 \\
\hline Radiation wavelength $[\mu \mathrm{m}]$ & $\sim 14$ \\
Power gain length $(2.2 \mathrm{nC})[\mathrm{cm}]$ & $\sim 14$
\end{tabular}

In this paper we perform theoretical analysis of the high gain SASE FEL experiment [1]. The analysis is based on the results produced by a three-dimensional, time-dependent FEL simulation code S\&D-3T [2]. Parameters of the electron beam and of the undulator [1] have been introduced "as is" as the input parameters for the numerical simulation code. It is shown that the obtained experimental results are in good agreement with the results of numerical calculations. Statistical simulations of the energy fluctuations in the radiation pulse performed over several thousands shots give the result identical to the experimental one. Namely, fluctuations of the energy in the radiation pulse follow gamma distribution with the value of parameter $M \simeq 8$. We also predict specific behaviour of another characteristics of the SASE FEL which can be measured during further experiments.

\section{Region of physical parameters}

We begin our study with analysis of physical effects influencing the operation of the FEL amplifier. Within the scope of the three-dimensional theory of the FEL amplifier we obtain that all the important physical effects are connected with the corresponding dimensionless parameters defining the power of the effect. These parameters are the diffraction parameter $B$, the space charge parameter $\hat{\Lambda}_{\mathrm{p}}^{2}$, the parameter of the longitudinal velocity spread $\hat{\Lambda}_{\mathrm{T}}^{2}$ and the efficiency parameter $\rho_{3 \mathrm{D}}[23,24,25]$ :

$$
\begin{aligned}
B & =2 \Gamma \sigma_{\mathrm{r}}^{2} \omega / c \\
\hat{\Lambda}_{\mathrm{p}}^{2} & =\Lambda_{\mathrm{p}}^{2} / \Gamma^{2}=4 c^{2}\left(\theta_{\mathrm{s}} \sigma_{\mathrm{r}} \omega A_{J J}\right)^{-2} \\
\hat{\Lambda}_{\mathrm{T}}^{2} & =\Lambda_{\mathrm{T}}^{2} / \Gamma^{2}=\left(\sigma_{\mathrm{E}}^{2} / \mathcal{E}_{0}^{2}+\gamma_{\mathrm{z}}^{4} \sigma_{\theta}^{4} / 4\right) / \rho_{3 \mathrm{D}}^{2} \\
\rho_{3 \mathrm{D}} & =c \gamma_{\mathrm{z}}^{2} \Gamma / \omega .
\end{aligned}
$$

The gain parameter $\Gamma$ defines the scale of the field gain and is defined as 


$$
\Gamma=\left[I A_{J J}^{2} \omega^{2} \theta_{\mathrm{s}}^{2} /\left(2 I_{\mathrm{A}} c^{2} \gamma_{\mathrm{z}}^{2} \gamma\right)\right]^{1 / 2},
$$

where $\omega=2 \pi c / \lambda$ is the frequency of the radiation field and $I_{\mathrm{A}}=m_{\mathrm{e}} c^{3} / e$. To be specific, we have written all the formulae for the case of the gaussian distribution of the electrons in the transverse phase space with the RMS radius and the RMS angle spread given by $\sigma_{\mathrm{r}}=\sqrt{\epsilon_{\mathrm{n}} \beta / \gamma}$ and $\sigma_{\theta}=\sqrt{\epsilon_{\mathrm{n}} / \beta \gamma}$, where $\epsilon_{\mathrm{n}}$ is rms normalized emittance, $\beta$ is focusing beta function and $\gamma=\mathcal{E}_{0} / m_{\mathrm{e}} c^{2}$ is relativistic factor. Energy spread in the electron beam is assumed to be gaussian with the rms deviation $\sigma_{\mathrm{E}}$. Undulator is assumed to be planar with amplitude of the magnetic field $H_{\mathrm{w}}$ and period $\lambda_{\mathrm{w}}$. The undulator parameter $K$, the angle of electron oscillations $\theta_{\mathrm{s}}$, the longitudinal relativistic factor $\gamma_{\mathrm{z}}$ and the factor $A_{J J}$ are defined as follows: $K=e H_{\mathrm{w}} \lambda_{\mathrm{w}} / 2 \pi m_{\mathrm{e}} c^{2}, \theta_{\mathrm{s}}=K / \gamma, \gamma_{\mathrm{z}}^{2}=\gamma^{2} /\left(1+K^{2} / 2\right)$ and $A_{J J}=J_{0}\left(K^{2} /\left(4+2 K^{2}\right)\right)-J_{1}\left(K^{2} /\left(4+2 K^{2}\right)\right)$, where $J_{0}$ and $J_{1}$ are the Bessel functions.

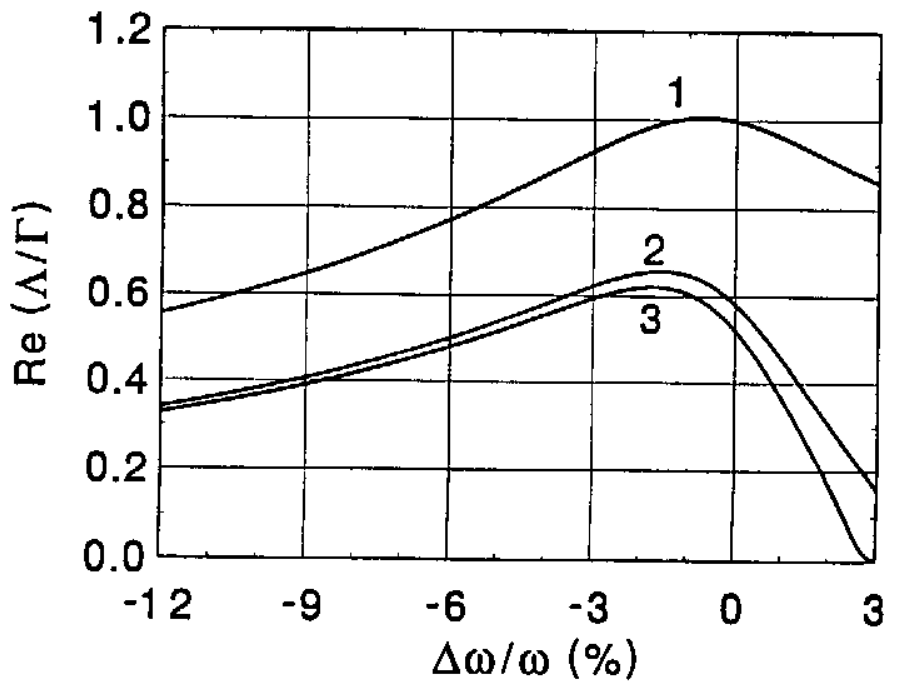

Fig. 1. The field gain versus the frequency deviation from the resonance value. Calculations have been performed in the steady-state approximation. Curve 1 is calculated with the only diffraction effects taken into account, curve 2 includes also the space charge effects, and curve 3 is calculated taking into account all the effects (diffraction, space charge and energy spread)

Calculation of the dimensionless parameters for the case of the operation of UCLA/LANL FEL at $Q=2.2 \mathrm{nC}$ gives the following values: $\Gamma^{-1}=19.3 \mathrm{~cm}, B=0.11, \hat{\Lambda}_{\mathrm{p}}^{2}=1.8$, $\hat{\Lambda}_{\mathrm{T}}^{2}=0.02, \rho_{3 \mathrm{D}}=0.0085$. It is seen that the main physical effects defining the operation of the FEL amplifier are the diffraction effects and the space charge effects. Numerical solution of the corresponding eigenvalue equation $[24,25]$ confirms this simple physical consideration (see Fig. 1). It is seen from the plot in Fig. 1 that the field gain length should be about $30 \mathrm{~cm}$ and is influenced mainly by the diffraction effects and the space charge effects. Also, analysis of the imaginary value of the eigenvalue shows that the slippage effect will be suppressed by a factor of four with respect to kinematic slippage due to the fact that the group velocity of the amplified wave, $\partial \omega / \partial k$, is less than the velocity of light $c$. Using the results of the 1-D approximation we can also estimate the effective power of shot noise at the undulator entrance $[13,19]$ : 


$$
\hat{\eta}=\frac{\sqrt{4 \pi} \rho^{2} P_{\mathrm{b}}}{3 \sqrt{\sqrt{3} \hat{z}} N_{\lambda}} \simeq 40 \mathrm{~mW},
$$

where $P_{\mathrm{b}}$ is the power in the electron beam, $N_{\lambda}$ is the number of electrons per radiation wavelength, $\hat{z}$ is the undulator length normalized to the gain parameter $\Gamma$ as $\hat{z}=z \Gamma B^{-1 / 3}$, and $\rho$ is the efficiency parameter of the one-dimensional approximation $[5]\left(\rho=\rho_{3 D} B^{-1 / 3}\right)$. Taking into account the value of the field gain (see Fig. 1) we obtain that the peak output power at the undulator exit should be about $8 \mathrm{~kW}$ and the energy in the radiation pulse should be of about $40 \mathrm{~nJ}$. We will show below with the results of the numerical simulation code that this estimation is very close to the actual value. Such a good accuracy of the estimation is due to a small value of the diffraction parameter $B$ [19].

\section{Numerical simulation code}

Numerical simulations have been performed with three-dimensional, time-dependent FEL simulation code S\&D-3T [2]. The code is realized in two versions: linear and nonlinear. In the linear mode of operation the evolution of the beam distribution function is described by the kinetic equation which accelerates significantly the speed of calculation. Nonlinear mode of operation is simulated with conventional method of macroparticles. The equations of motion (kinetic equation or equations for macroparticle motion) and Maxwell's equations are solved simultaneously taking into account the slippage effect. Radiation fields are calculated using integral solution of Maxwell's equations (in the form of retarded potentials). Such physically transparent representation of the radiation field can be simply incorporated into time-dependent simulations. Special technique has been developed for fast calculations of the retarded potentials reducing drastically required CPU time. As a result, the developed code allows one to use personal computer for time-dependent simulations. The code allows one to simulate the radiation from the electron bunch of any transverse and longitudinal bunch shape; to simulate simultaneously external seed with superimposed shot noise in the electron beam; to take into account energy spread in the electron beam and the space charge fields; and to simulate high-gain, high-efficiency FEL amplifier with tapered undulator. It is important, that in the developed code there are no memory limitations and the electron bunch of any length can be simulated. The accuracy of simulations with the developed code can be controlled, since it has been tested thoroughly in a steady-state limit with analytical solutions and with existent steady-state codes. 


\section{Numerical analysis of the experiment}

To calculate averaged characteristics of the FEL amplifier we performed several thousands statistically independent runs. Input data for the numerical simulation code are the value of the undulator period and the peak field, the value of the bunch charge, the value of the RMS bunch length and the RMS bunch radius. When performing simulations we used two models of the axial profile of the bunch current, a gaussian one

$$
I(z)=\frac{Q c}{\sqrt{2 \pi} \sigma_{\mathrm{z}}} \exp \left[-\frac{z^{2}}{2 \sigma_{\mathrm{z}}^{2}}\right],
$$

with $\sigma_{\mathrm{z}}=\sigma_{\mathrm{z}}^{\mathrm{HWHM}} / \sqrt{2 \ln 2}$, and a parabolic one:

$$
I(z)=\frac{3 Q c}{4 \sigma_{\mathrm{z}}}\left[1-\frac{z^{2}}{\sigma_{\mathrm{z}}^{2}}\right], \quad|z|<\sigma_{\mathrm{z}},
$$

with $\sigma_{\mathrm{z}}=\sqrt{2} \sigma_{\mathrm{z}}^{\mathrm{HWHM}}$. Transverse distribution of the beam current density assumed to be gaussian:

$$
j(z, r)=\frac{I(z)}{2 \pi \sigma_{\mathbf{r}}^{2}} \exp \left[-\frac{r^{2}}{2 \sigma_{\mathbf{r}}^{2}}\right]
$$

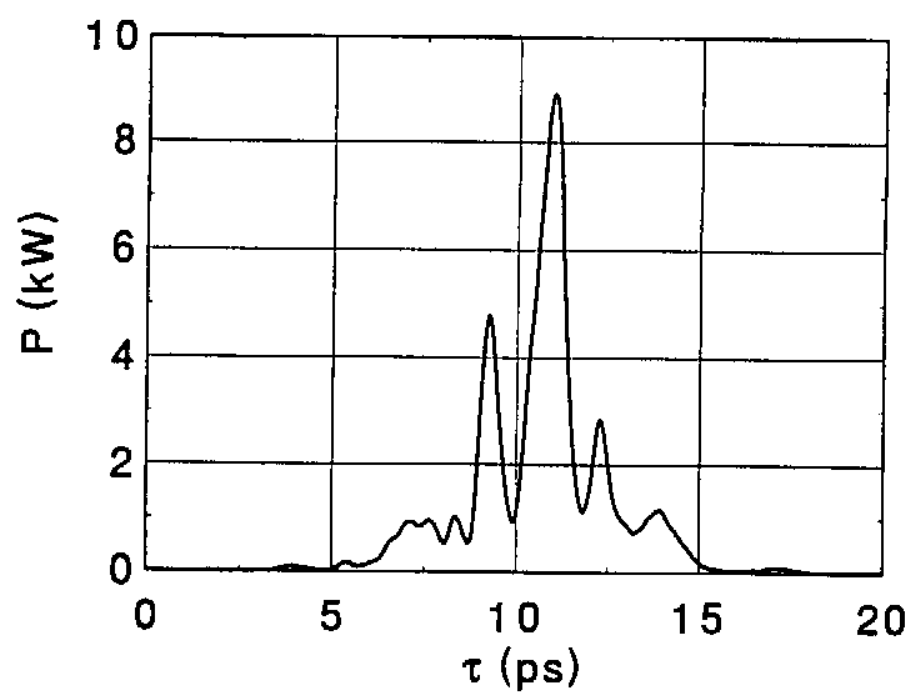

Fig. 2. Typical time structure of the radiation pulse at the undulator exit. Charge in the electron bunch is $2.2 \mathrm{nC}$

with $\sigma_{\mathrm{r}}=\sigma_{\mathrm{r}}^{\mathrm{HWHM}} / \sqrt{2 \ln 2}$. HWHM values are defined by fitting formulae presented in ref. [1]: $\sigma_{\mathrm{z}, \mathrm{r}}^{\mathrm{HWHM}}=\sqrt{a^{2}+(b Q)^{2}}$. Parameters for the spot size are $a=120 \mu \mathrm{m}$, $b=38 \mu \mathrm{m} / \mathrm{nC}$, and for the pulse length $a=3 \mathrm{ps}, b=2.2 \mathrm{ps} / \mathrm{nC}$.

In Fig. 2 we present typical time structure of the radiation pulse at the undulator exit at the value of the bunch charge of $2.2 \mathrm{nC}$. Averaging over one thousand of independent shots gives the value of the averaged radiation pulse shape (see Fig. 3). In particular, analysis of the latter plot allows one to obtain the value of "effective" shot noise power 


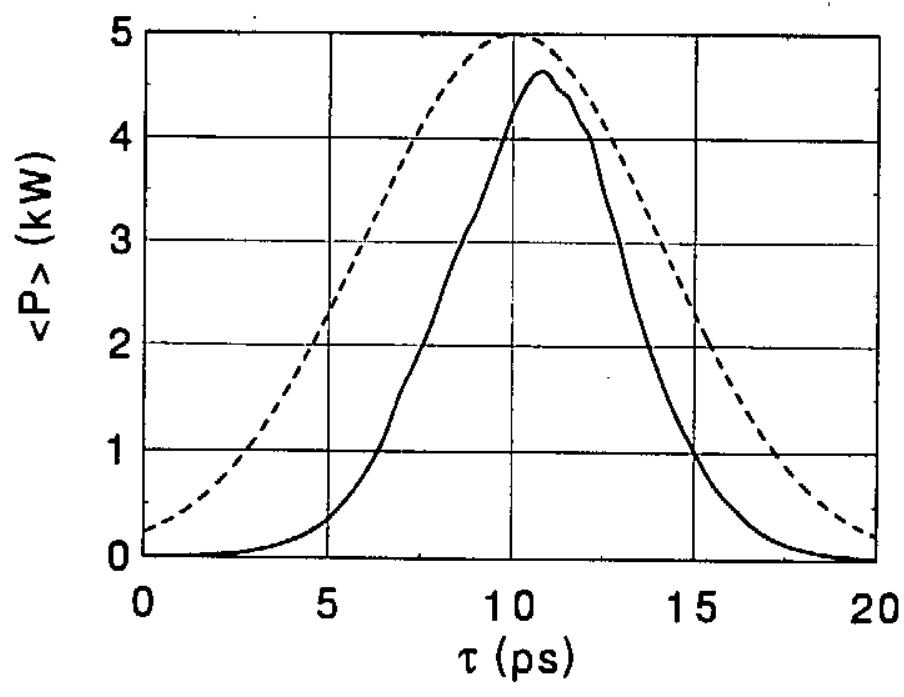

Fig. 3. Time structure of the radiation pulse at the undulator exit averaged over 1000 statistically independent runs. Charge in the electron bunch is $2.2 \mathrm{nC}$. Dashed line presents axial profile of the beam current

at the undulator entrance to be used in the steady-state codes for obtaining approximate value of the average output power $[13,19]$. The value of the "effective" power of shot noise is of about $20 \mathrm{~mW}$ in the case under study which is close to the 1-D estimation presented above. It is seen also from Fig. 3 that the slippage of the radiation with respect to the electron bunch is significantly smaller than kinematic one. This is connected with the fact that the group velocity of the spikes, $\partial \omega / \partial k$, is less than the velocity of light $c$ as it has been mentioned in section 2 .

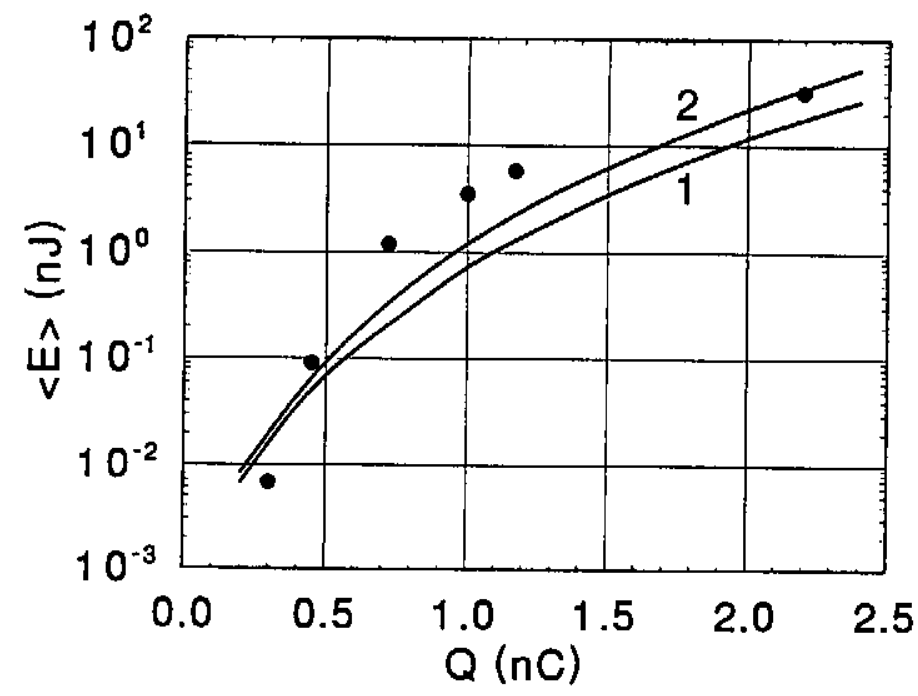

Fig. 4. Dependence of the averaged energy in the radiation pulse versus the bunch charge. Curves 1 and 2 correspond to the gaussian and the parabolic axial beam profiles. The circles are experimental results [1]

In Fig. 4 we present comparison of experimental and simulation results for different values of the charge. Fig. 5 presents the dependency of the energy in the radiation pulse on the transverse and longitudinal beam sizes at a fixed value of the bunch charge of $1 \mathrm{nC}$. It is seen that within uncertainties in measuring these values $[1,26,27]$, numerical and experimental results agree rather well. One can also obtain unusual behaviour of 


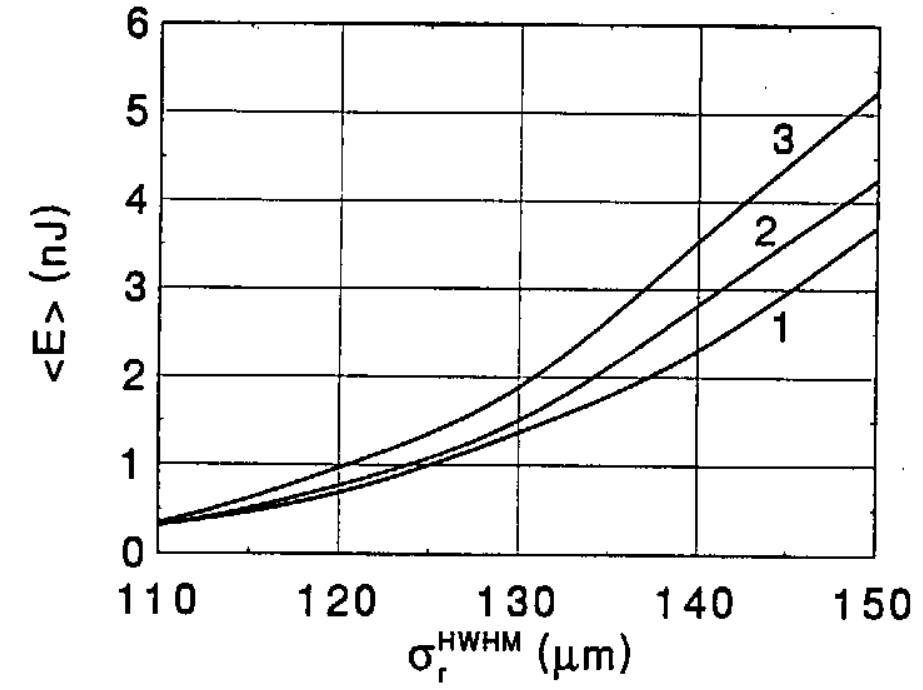

Fig. 5. Dependence of the averaged energy in the radiation pulse on the transverse bunch size. Curves 1,2 and 3 correspond to the values of the longitudinal HWHM bunch size $\sigma_{\mathrm{z}}^{\text {HWHM }}$ of $3.9 \mathrm{ps}, 3.7 \mathrm{ps}$ and $3.5 \mathrm{ps}$, respectively. The bunch charge is $1 \mathrm{nC}$

the energy in the radiation pulse on the value of the transverse beam size. Namely, the radiation energy is increased at the increase of the transverse beam size. This effect is connected with the fact which we have mentioned in section 2 that UCLA/LANL FEL amplifier operates in the regime of a strong influence of the space charge effects. In the case under study the diffraction parameter $B$ is much less than unity, and increasing of the beam size results only in logarithmic decreasing of the field gain due to diffraction effects $[23,24]$. On the other hand, there is strong influence of the space charge fields. Increasing of the transverse beam size leads to quadratic decrease of the space charge parameter which results in the increase of the field gain. In the region of parameters traced in Fig. 5 this effect dominates above the diffraction effects. As a result, the field gain and the energy in the radiation pulse grow with the increase of the transverse size of the electron beam. Calculations show that such a tendency will take place up to the value of diffraction parameter $B \sim 0.3$. Above this pont the space charge effect becomes to be a small perturbation to the FEL process and increase of the transverse beam size will lead to the decrease of the field gain. In other words this means that maximal gain in the UCLA/LANL SASE FEL should occur at a larger value of the focusing beta function than that used in the experiment. Similar problem has been studied earlier (see, e.g. refs. $[28,29])$.

So, we see that there is good agreement between measurements of the energy in the radiation pulse and the simulation results. Indeed, in all the region of the charge of the electron beam (see Fig. 4), the relative difference recalculated in the units of the gain length is less than one power gain length. Taking into account good agreement of the energy in the radiation pulse, we can expect more better agreement with the probability distribution of the energy in the radiation pulse. We performed the corresponding simulations at the value of the bunch charge of $2.2 \mathrm{nC}$. The first set of runs has been 


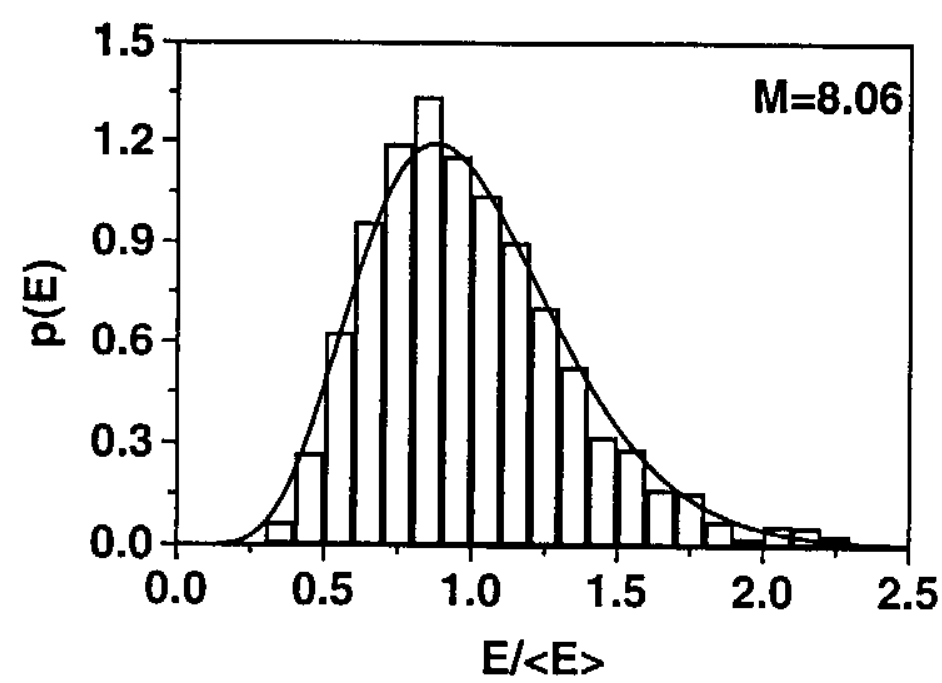

Fig. 6. Probability distribution of the energy in the radiation pulse at the bunch charge of $2.2 \mathrm{nC}$ calculated over 2400 statistically independent runs. Solid curve presents gamma distribution with $M=8.06$

performed at fixed values of the bunch charge, of the transverse beam size and of the longitudinal beam size. Simulations with the gaussian and the parabolic bunch profiles give very close results, namely the probability distribution of the energy in the radiation pulse is quite close to the gamma distribution with $\sigma \simeq 30 \%$ and $M \simeq 11.5$. On the other hand, experimental result gives the value of the fluctuations of about $\sigma \simeq 37 \%$. Such a visible difference indicates that shot-to-shot fluctuations of the beam parameters contribute to the fluctuations of the radiation energy. To take these fluctuations into account we performed 2400 statistically independent runs. During each run the fluctuations have been introduced in the following limits: $\pm 0.75 \%$ for the bunch charge, $\pm 5.5 \%$ for the transverse beam size and $\pm 6 \%$ for the bunch length [1]. The results of the simulations are shown in Fig. 6. The probability distribution of the radiation energy follows the gamma distribution with $\sigma \simeq 35 \%$ and $M \simeq 8$. This result is in a good agreement with the experiment. May be, agreement could be even better if we take into account the noise in the detector itself, but these experimental data are not available in paper [1].

\section{Some predictions for future experiment}

So, we see that there is good agreement between numerical and experimental results. This allows us to predict some properties of the UCLA/LANL SASE FEL which can be measured in the future experiments. In Fig. 7 we present normalized spectrum averaged over large number of shots. It is seen that the value of the maximum is shifted significantly with respect to the resonance value. Partially this is consequence of diffraction effects, of a finite value of the gain, and of the space charge effects. All these effects shift the maximum of the spectrum in the same direction.

The directivity diagram of the radiation intensity is one of the important characteristics of FEL amplifier. At a large distance $L$ from the amplifier exit the output radiation has 

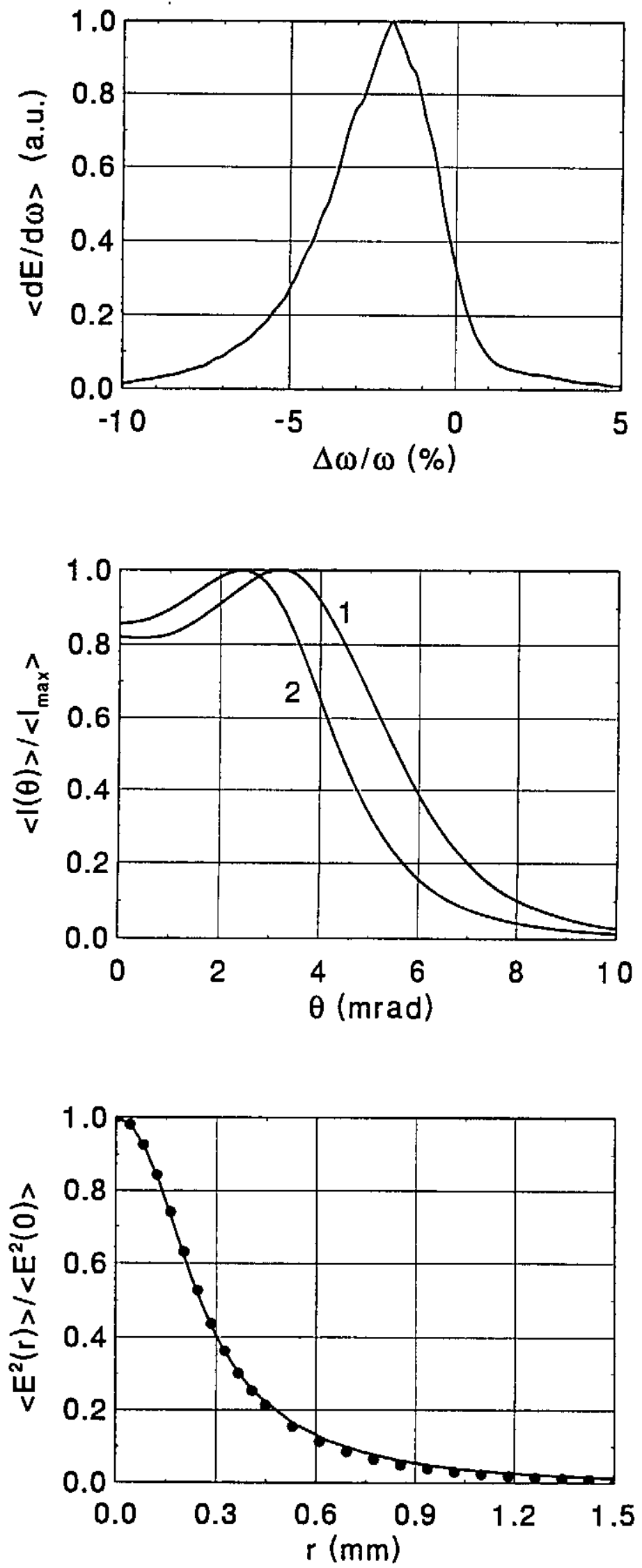

Fig. 7. Radiation spectrum averaged over 1000 statistically independent runs. Bunch charge is $2.2 \mathrm{nC}$
Fig. 8. Directivity diagram of the radiation intensity in far zone averaged over a large number of shots (curve 1). Curve 2 presents directivity diagram of the FEL amplifier operating in the steady state regime at tuning on the maximum of the gain. Charge in the bunch is $2.2 \mathrm{nC}$
Fig. 9. Radial distribution of the radiation intensity at the undulator exit averaged over a large number of shots. The circles present the corresponding distribution for the FEL amplifier operating in the steady state regime at tuning on the maximum of the gain. Charge in the bunch is $2.2 \mathrm{nC}$ 
the form of a spherical wave. In the axisymmetric case the radiation intensity, $I=|E|^{2}$, normalized to maximal value, is universal function of the $\theta=r / L$, where $r$ is observation point in far zone. In Fig. 8 we present the directivity diagram of the radiation intensity, $I(\theta) / I_{\max }$ averaged over many shots. One can obtain that the maximum of the directivity diagram occurs at a nonzero observation angle. On the other hand, distribution of the radiation intensity at the undulator exit along the transverse coordinate is a smooth one (see Fig. 9). Such an unusual behaviour of the directivity diagram is a consequence of the space charge influence. For comparison we present the corresponding directivity diagram for the steady-state FEL amplifier tuned to the maximum of the field gain. We hope that experimental resolution will be sufficient to resolve this fine effect.

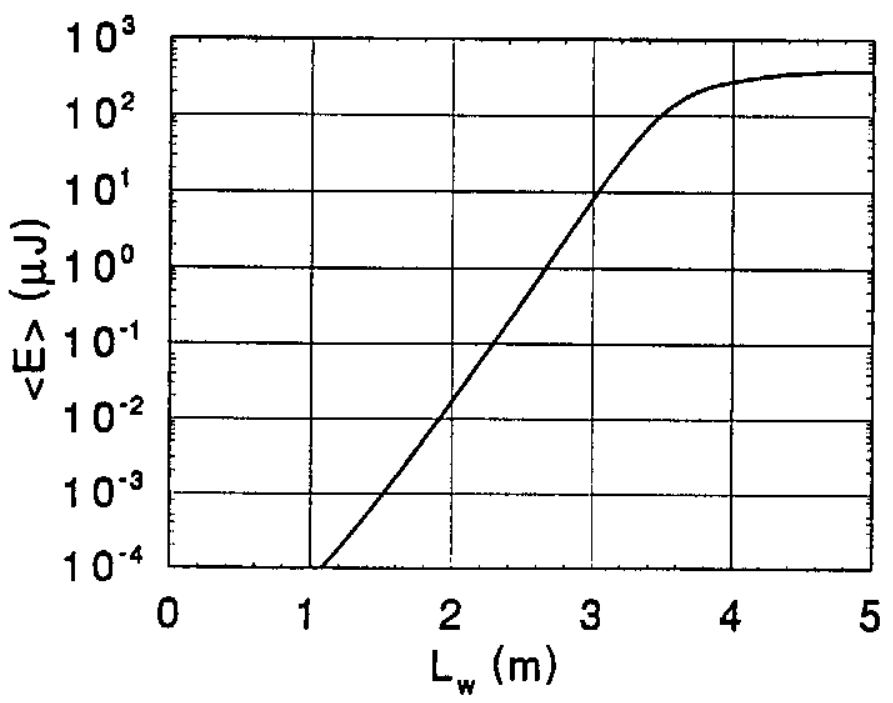

Fig. 10. The dependence of the averaged energy in the radiation pulse over the length of the undulator. Simulations have been performed with nonlinear simulation code. Charge in the bunch is $2.2 \mathrm{nC}$

Finally, in Fig. 10 we present the dependence of the energy in the radiation pulse as a function of the undulator length. Calculations have been performed with nonlinear simulation code [2]. It is seen that saturation in UCLA/LANL SASE FEL will occur at the undulator length of about $4 \mathrm{~m}$. Installation of an additional undulator module of $2 \mathrm{~m}$ length will allow to perform investigations of the SASE FEL operating exactly at the saturation point.

\section{Conclusion}

In conclusion to this paper we should like to notice that there is no doubt that UCLA/LANL experiment [1] is a proof-of-principle of a high-gain SASE FEL. Despite it has been performed at a relatively long wavelength, the physics of its operation is described with the same equations as future VUV and X-ray SASE FELs. All the simulations presented in this paper have been performed with the simulation code developed 
for simulation of short-wavelength SASE FELs. It is seen that there is good agreement between theoretical predictions and experimental results, which forms reliable base for future design of short-wavelength SASE FELs.

\section{Acknowledgments}

We are extremely grateful to C. Pellegrini and A. Varfolomeev for providing us with experimental results and fruitful discussions. We wish to thank J. Feldhaus, J. Krzywinski, G. Materlik, T. Möller, J. Pflüger, S. Reiche, J. Roßbach and J.R. Schneider for many useful discussions. 


\section{References}

1. M. Hogan, C. Pellegrini, J. Rosenzweig, A. Anderson, P. Frigola, A. Tremaine, C. Fortgang, D. Nguyen, R. Sheffield, J. Kinross-Wright, A. Varfolomeev, A.A. Varfolomeev, S. Tolmachev, R. Carr, "Measurement of Gain Larger Than $10^{5}$ at $12 \mu \mathrm{m}$ in a SASE FEL ${ }^{n}$, submitted to Physical Review Letters.

2. E.L. Saldin, E.A. Schneidmiller and M.V. Yurkov, "Three dimensional, time-dependent FEL simulation code S\&D-3T".

3. Ya.S. Derbenev, A.M. Kondratenko and E.L. Saldin, Nucl. Instrum. and Methods 193(1982)415.

4. J.B. Murphy and C. Pellegrini, Nucl. Instrum. and Methods A237(1985)159.

5. R. Bonifacio, C. Pellegrini and L. Narducci, Opt. Commun. 50(1984)373.

6. R. Tatchyn et al., Nucl. Instrum. and Methods A375(1996)274.

7. "A VUV Free Electron Laser at the TESLA Test Facility: Conceptual Design Report", DESY Print TESLAFEL 95-03, Hamburg, DESY, 1995.

8. J. Rossbach et al., Nucl. Instrum. and Methods A375(1996)269.

9. "Conceptual Design of a $500 \mathrm{GeV}$ e+e- Linear Collider with Integrated X-ray Laser Facility" (Editors R.Brinkmann, G. Materlik, J. Rossbach, A. Wagner), DESY 97-048, Hamburg, 1997.

10. I. Ben-Zvi, Nucl. Instrum. and Methods A375(1996)269.

11. S. Suzuki, K. Yanagida, T. Taniuchi, Y. Kishimoto, A. Mizuno, H. Abe, H. Yoshikawa and H. Yokomizo, AIP Conference Proceedings 413(1997)121.

12. A.M. Kondratenko and E.L. Saldin, Part. Acc. 10(1980)207.

13. K.J. Kim, Nucl. Instrum. and Methods A250(1986)396.

14. K.J. Kim, Phys. Rev. Lett. 57(1986)1871.

15. J.M. Wang and L.H. Yu, Nucl. Instrum. and Methods A250(1986)484.

16. S. Krinsky and L.H. Yu, Phys. Rev. A35(1987)3406.

17. R. Bonifacio et al., Phys. Rev. Lett. 73(1994)70.

18. E.L. Saldin, E.A. Schneidmiller and M.V. Yurkov, "Statistical Properties of Radiation from VUV and X-ray Free Electron Laser", DESY Print TESLA-FEL 97-02, April, 1997.

19. E.L. Saldin, E.A. Schneidmiller and M.V. Yurkov, Opt. Commun. 148(1998)383.

20. P. Pierini and W. Fawley, Nucl. Instrum. and Methods A375(1996)332.

21. W.M. Fawley, SPIE Proceedings 2988(1997)98.

22. W.M. Fawley, private communication.

23. E.L. Saldin, E.A. Schneidmiller and M.V. Yurkov, Phys. Rep. 260(1995)187.

24. E.L. Saldin, E.A. Schneidmiller and M.V. Yurkov, Opt. Commun. 97(1993)272.

25. E.L. Saldin, E.A. Schneidmiller and M.V. Yurkov, "Calculation with FS2R Code of $6 \mathrm{~nm}$ and $70 \mathrm{~nm}$ Options of SASE FEL at the TESLA Test Facility", DESY Print May 1995, TESLA-FEL 95-02, Hamburg, DESY, 1995.

26. R.L. Sheffield, R.H. Austin, K.C.D. Chan, S.M. Gierman, J. Kinross-Wright, S.H. Kong, D.C. Nguyen, S.J. Russel and C.A. Timmer, Nucl. Instrum. and Methods A341(1994)371.

27. S.H. Kong, J. Kinross-Wright, D.C. Nguyen, R.L. Sheffield and M.E. Weber, Nucl. Instrum. and Methods A358(1995) 284.

28. E.L. Saldin, V.P. Sarantsev, E.A. Schneidmiller and M.V. Yurkov, "FEL based photon collider of TeV energy range", Preprint JINR E9-94-70, Dubna, 1994.

29. E.L. Saldin, E.A. Schneidmiller and M.V. Yurkov, Proceedings of the Fifth European Particle Accelerator Conference, Vol.1, pp. 471-473 (Institute of Physics Publishing, Bristol, 1996).

30. W. Brefeld, B. Faatz, Y.M. Nikitina, J. Pflüger, P. Pierini, J. Roßbach, E.L. Saldin, E.A. Schnejdmiller and M.V. Yurkov, Nucl. Instrum. and Methods A393(1997)119. 